\title{
Loading Capacity Analysis of Hydrostatic Oil Pad Based on Fluid- structure Coupling
}

\author{
Yongsheng Zhao ${ }^{\mathrm{a}}$, Haiming Zhang ${ }^{\mathrm{b}}$, Weizhe $\mathrm{Yu}^{\mathrm{c}}$ \\ Beijing University of Technology, Beijing 100124, China \\ ayszhao@bjut.edu.cn, bhbkjzhm@163.com, wzyu@bjut.edu.cn
}

Keywords: hydrostatic oil pad, Loading Capacity, Reynolds equation, Fluid-structure Coupling, finite difference method

\begin{abstract}
Hydrostatic guideway is the key component of heavy-duty CNC machine tool feed system. The working performance of the hydrostatic guideway have great influence to the accuracy of machine tool. In order to analysis the performance of the oil pad, a calculation method based on Reynolds equation and the thin plate deformation equation, considering the fluid-structure coupling, is established. The influence of film thickness, oil supply parameter and inclination to the performance of the oil pad are discussed. It is shows that loading capacity declined compared with the situation which is not considering the fluid-structure coupling.
\end{abstract}

\section{Introduction}

Heavy-duty CNC machine tool is the important component of defense industry, aerospace, shipbuilding, energy, transportation, metallurgy and mechanical engineering. As the improvement of technology, the requirement to the heavy-duty $\mathrm{CNC}$ machine tools are high-precision and high loading-capacity. Hydrostatic guideway is the key component of heavy heavy-duty CNC machine tool feed system. Because of the advantage of high-precision, high loading-capacity and low friction, hydrostatic guideway is widely used between the two moving part in machine tool. Therefore the work performance of the hydrostatic guide have great influence to the accuracy of machine tool. Numerous studies on hydrostatic bearing can be found in literature, where the researchers have paid attention to the various aspects of the characteristics of hydrostatic guideways.

N.D.Manring [1] analyzed the impact of linear deformation on the flow rate and the load carrying capacity of the stationary hydrostatic bearing. Jaw-Ren Lin [2] analyzed the effect of surface roughness on the dynamic stiffness and damping characteristics of compensated hydrostatic thrust bearings. Bouyer [3] combined the Reynolds equation with energy equation to make a thermoelastohydrodynamic analysis on a plain journal bearing with a single groove under steady load. And the bearing deformation was also taken into account. WANG [4-5] studied the influence of oil film, the flow rate to the stiffness of the hydrostatic thrust bearing by theory and experiment. KAZAMA [6-7] proposed an optimum design method for the elastic deformation of the oil lubricated nonmetallic bearing. Experimental study has proved that the change of hydraulic pressure can cause the elastic deformation of the structure by PANG [8].

In summary, the analysis of fluid-structure interaction is usually for a specific object. However, there is a lack of research on the general working conditions of static hydrostatic guideway rail. In this paper, according to the general conditions of the static pressure guide rail, the oil film characteristics analysis equations and the guide rail surface deformation equation are constructed, and the connection between them is constructed by the oil film thickness, and the fluid-structure coupling characteristic analysis model is established. The conditions of different oil supply parameter, different oil film thickness, and different guide surface tilt are analyzed.

\section{Background}

The structure diagram of hydrostatic oil pad is depict in Fig. 1.Rectangle hydrostatic oil pad is widely used in straight hydrostatic guideway of heavy duty machine tools. The oil pads are along the guide rail. Pressurized oil get into the Rectangle oil pad from the oil feeding system. As is shown in 
Fig. 1, the length of the oil pad is L, the width of the oil pad is B, the length of the oil pocket is 1, the width of oil the pocket is $b$, the film thickness is $h$ and the inclination angle of the railway surface is $\emptyset$.
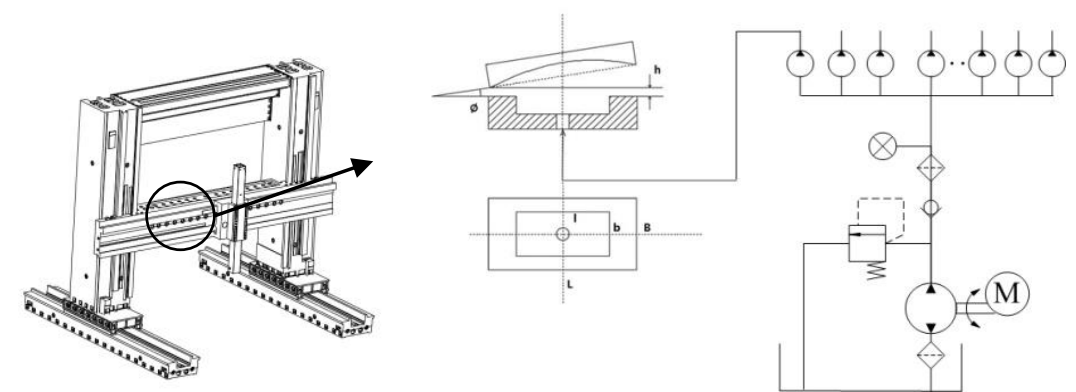

Fig. 1 The structure diagram of hydrostatic oil pad

Because of the overturning moment caused by the gravity of the slide carriage and the ram, the thickness of the oil pad between the column and the beam changes. And the force from the oil film to the railway surface makes the surface out of shape. The railway deformation and the film thickness changing have a great influence to the loading capacity of the hydrostatic railway.

\section{The loading capacity analysis model of Rectangle single hydrostatic oil pad based on Fluid- structure Coupling}

The speed of straight hydrostatic railway in Heavy-duty CNC machine tool is always to be low. According to the assumptions of Reynolds equation, the variation of the viscosity and density could be neglect. Therefore the Reynolds equation for analyzing the rectangle single hydrostatic oil pad is written as

$$
\frac{\partial}{\partial x}\left(\frac{\rho h^{3}}{\eta} \frac{\partial p}{\partial x}\right)+\frac{\partial}{\partial y}\left(\frac{\rho h^{3}}{\eta} \frac{\partial p}{\partial y}\right)=6\left[\frac{\partial}{\partial x}(U \rho h)+\frac{\partial}{\partial y}(V \rho h)\right]
$$

In order to get the pressure distribution of the oil film, Finite difference method has been used to solve the Reynolds equation. Before solving the equation, the parameter should be nondimensionalized.

$$
\bar{p}=\frac{p}{p_{0}}, \overline{p_{0}}=1, \bar{x}=\frac{x}{L}, \bar{L}=1, \bar{y}=\frac{y}{B}, \bar{B}=1, \overline{\mathrm{h}}=\frac{h}{H_{0}}, \overline{H_{0}}=1, \overline{U_{x}}=\frac{U_{x}}{\frac{H_{0}^{2} p_{0}}{L \eta}}, \bar{q}=\frac{q}{\frac{H_{0}^{3} p_{0}}{\eta}}
$$

Where, $\mathrm{p}$ is pressure, $\mathrm{p}_{0}$ is pressure in the oil pocket, $\mathrm{q}$ is volumetric flow rate, $\mathrm{Ux}$ is velocity of $\mathrm{x}$ coordinate, $h$ is film thickness and $\eta$ is viscosity. In addition, $\bar{p}$ is dimensionless pressure, $\bar{x}$ is dimensionless size on $\mathrm{x}$ coordinate, $\overline{\mathrm{y}}$ is dimensionless size on y coordinate, $\overline{\mathrm{z}}$ is dimensionless size on $z$ coordinate, $\bar{q}$ is dimensionless flow rate, $\overline{U_{x}}$ is dimensionless velocity of $x$ coordinate and $\bar{h}$ is dimensionless film thickness. The pressure distribution of the oil film can be written as

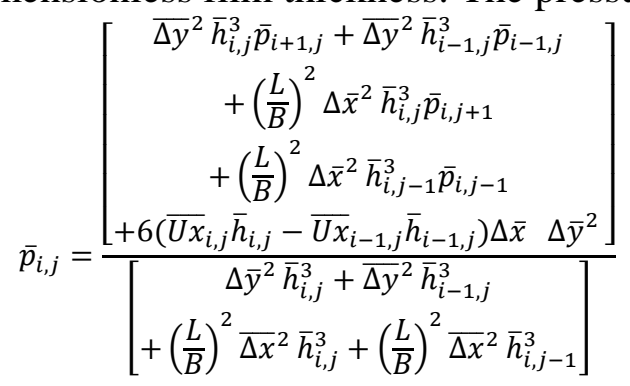

Therefore, the loading capacity of the oil film could be expressed as

$$
F=\frac{\bar{p}_{i, j} \cdot \bar{x}_{\text {step }} \cdot \bar{y}_{\text {step }} L B \eta}{\bar{q} h_{0}^{3}} \cdot Q
$$

Where $Q$ is the supply flow rate of the oil pad, $\bar{x}_{\text {step }}$ is step length of the $x$ coordinate, $\bar{y}_{\text {step }}$ is step length of the $y$ coordinate, $i$ and $j$ are numerical counts of the elements on $\mathrm{x}$ and $\mathrm{y}$ coordinates respectively. Discrete nodes of FDM are shown in Fig 2. 


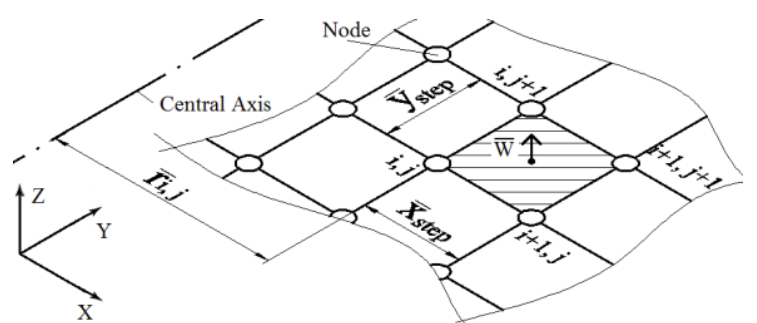

Fig.2. Discrete nodes of FDM

According to the basic principle of elastic mechanics, the deformation of the rail way surface can be written as

$$
\omega=\omega(\mathrm{x}, \mathrm{y})
$$

Where $\omega(\mathrm{x}, \mathrm{y})$ is the value of the deformation in $\mathrm{Z}$ direction, $\mathrm{x}$ and $\mathrm{y}$ is the same coordinate system as when solving the Reynolds equation. Take any element in the railway surface, The stress and strain in the three directions of $\mathrm{X}, \mathrm{Y}$ and $\mathrm{Z}$ are expressed as

$$
\begin{aligned}
& \sigma_{x}=-\frac{E}{1-\mu^{2}} \mathrm{z}\left(\frac{\partial^{2} \omega}{\partial x^{2}}+\mu \frac{\partial^{2} \omega}{\partial y^{2}}\right) \\
& \sigma_{y}=-\frac{E}{1-\mu^{2}} \mathrm{z}\left(\frac{\partial^{2} \omega}{\partial y^{2}}+\mu \frac{\partial^{2} \omega}{\partial x^{2}}\right) \\
& \tau_{x y}=-\frac{E}{1+\mu} z \frac{\partial^{2} \omega}{\partial x y}
\end{aligned}
$$

Simplify the stress to the middle plane of the railway:

$$
\begin{aligned}
& M_{x}=\int_{-\frac{h}{2}}^{\frac{h}{2}} z \sigma_{x} d z=-D\left(\frac{\partial^{2} \omega}{\partial x^{2}}+\mu \frac{\partial^{2} \omega}{\partial y^{2}}\right) \\
& M_{y}=\int_{-\frac{h}{2}}^{\frac{h}{2}} z \sigma_{y} d z=-D\left(\frac{\partial^{2} \omega}{\partial y^{2}}+\mu \frac{\partial^{2} \omega}{\partial x^{2}}\right) \\
& M_{x y}=M_{y x}=\int_{-\frac{h}{2}}^{\frac{h}{2}} z \tau_{x y} d z=-D(1-\mu) \frac{\partial^{2} \omega}{\partial x y}
\end{aligned}
$$

Where $\mathrm{D}$ is the bending stiffness of the railway, $\mathrm{D}=\frac{\mathrm{EH}^{3}}{12\left(1-\mu^{2}\right)} . \mathrm{H}$ is the thickness of the railway surface.Fig. 3 showns the Micro force diagram of the middle plane, the force balance equation is written as

$$
\begin{aligned}
& F_{S x}=\frac{\partial M_{x}}{\partial x}+\frac{\partial M_{x y}}{\partial y} \\
& F_{S y}=\frac{\partial M_{x y}}{\partial x}+\frac{\partial M_{y}}{\partial y} \\
& \frac{\partial F_{S x}}{\partial x}+\frac{\partial F_{S y}}{\partial y}+q=0
\end{aligned}
$$

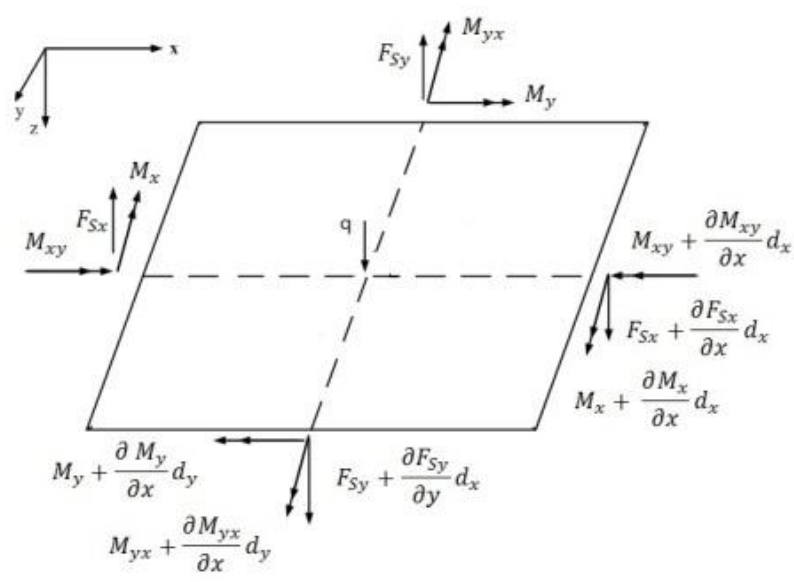

Fig.3 Micro force diagram of the middle plane 
Replace $\sigma_{\mathrm{z}}$ with $\omega$,the force balance equation is expressed as

$$
\frac{E h^{3}}{12\left(1-\mu^{2}\right)}\left(\frac{\partial^{4} \omega}{\partial x^{4}}+\frac{\partial^{4} \omega}{\partial y^{4}}+\frac{\partial^{4} \omega}{\partial z^{4}}\right)=q
$$

Where the concentrated forces is q. In order to determine the thickness of oil film, the boundary condition of the guide rail is simply supported. the length of the rail way surface is L, the width of the rail way surface is $\mathrm{B}$. The boundary condition can be shown as

$$
\begin{aligned}
& x=0, \omega=0, \frac{\partial^{2} \omega}{\partial x^{2}}=0 \\
& x=a, \omega=0, \frac{\partial^{2} \omega}{\partial x^{2}}=0 \\
& y=0, \omega=0, \frac{\partial^{2} \omega}{\partial y^{2}}=0 \\
& y=a, \omega=0, \frac{\partial^{2} \omega}{\partial y^{2}}=0
\end{aligned}
$$

If the coordinate of the concentrated forces is $(\mathrm{k}, \mathrm{l})$ and the force at the rest of the guide rail is 0 , the deformation of the coordinate $(\mathrm{x}, \mathrm{y})$ can be expressed as

$$
\omega=\frac{4 q}{\pi^{4} a b D} \sum_{m=1}^{\infty} \sum_{n=1}^{\infty} \frac{\sin \frac{m \pi k}{a} \sin \frac{n \pi l}{b}}{\left(\frac{m^{2}}{a^{2}}+\frac{n^{2}}{b^{2}}\right)^{2}} \sin \frac{m \pi x}{a} \sin \frac{n \pi y}{b}
$$

The Flow diagram of analysis model of rectangular hydrostatic pad based on Fluid-structure Coupling is shown in Fig.4. First the parameters of the oil pad, the thickness of the oil film and the supply flow rate should be determined. Then the pressure distribution of the oil film can be calculated with equation (2). The railway surface deformation caused by all pressure point could be calculated using equation (10). The new film thickness could be get by adding the old film thickness and the deformation of the surface. If the difference between the new and old film thickness matches the error tolerance, the loading capacity of the oil pad should be calculated with equation (3). If the difference does not matches the error tolerance, the pressure distribution should be calculated again using the new film thickness.

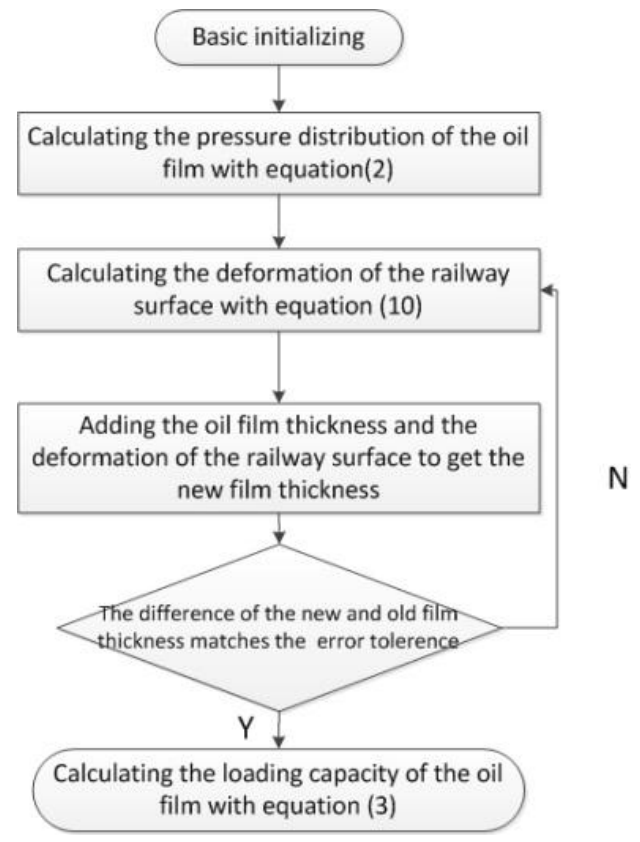

Fig.4 Flow diagram of analysis model of rectangular hydrostatic pad

\section{Results and discussions}

Based on the fluid solid coupling, the performance of a rectangular single oil pad is analyzed, and the parameters of oil pad are shown in Table 1. 
Table1 Parameter table of the oil pad

\begin{tabular}{cccc}
\hline Parameter & value & Parameter & value \\
\hline oil pad length L $(\mathrm{mm})$ & 100 & oil pocket length $1(\mathrm{~mm})$ & 66 \\
oil pad width B $(\mathrm{mm})$ & 66 & oil pocket width b $(\mathrm{mm})$ & 33 \\
viscosity $($ pas $)$ & 0.06 & Poisson ratio & 0.26 \\
railway surface length a $(\mathrm{mm})$ & 100 & railway surface thickness H $(\mathrm{mm})$ & 5 \\
railway surface width b $(\mathrm{mm})$ & 66 & elastic modulus(MPa) & $2.5 \times 10^{5}$ \\
\hline
\end{tabular}

\section{1 film thickness impact on loading capability of single oil pad}

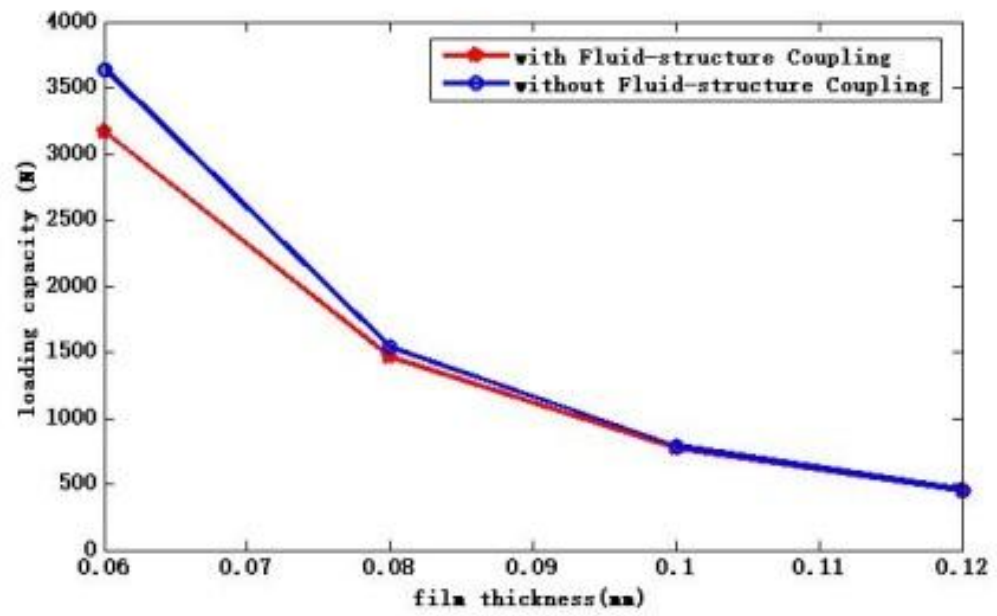

Fig.5 The variation of loading capacity with film thickness

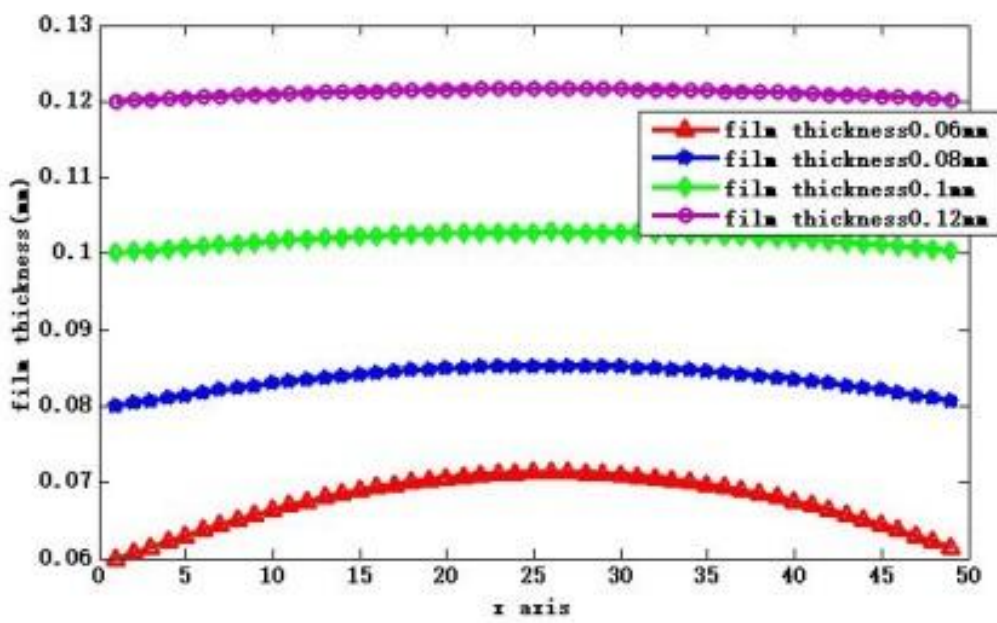

Fig.6 The variation of railway deformation with film thickness

Fig. 5 shows the variation of loading capacity with film thickness, while the supply flow rate is constant and the railway surface is Flat. It is indicate that the loading capacity reduces as the increasing of the film thickness. The loading capacity is lower considering the Fluid-structure Coupling compared with regarding the railway as rigid. And the gap between considering the Fluidstructure Coupling and regarding the railway as rigid becomes larger as the decrease of oil film thickness. The gap is $14.7 \%$, when the film thickness is $0.06 \mathrm{~mm}$. while the gap is $1.1 \%$, when the film thickness is $0.12 \mathrm{~mm}$. That is because the deformation of the railway surface increases as the decrease of oil film thickness. The variation of railway deformation with film thickness is shown in Fig.6.

\section{2 supply flow rate impact on loading capability of single oil pad}

Fig.7 shows the variation of loading capacity with supply flow rate, while the film thickness is $0.1 \mathrm{~mm}$ and the railway surface is Flat. It is indicate that the loading capacity increases as the increasing of supply flow rate. The loading capacity is lower considering the Fluid-structure Coupling compared with regarding the railway as rigid. And the gap between considering the Fluid-structure Coupling and regarding the railway as rigid becomes larger as the increasing of supply flow rate. The 
gap is $3.2 \%$, when the supply flow rate is $0.18 \mathrm{~L} / \mathrm{min}$. That is because the deformation of the railway surface increases as the increasing of supply flow rate. The variation of railway deformation with film thickness is shown in Fig.8.

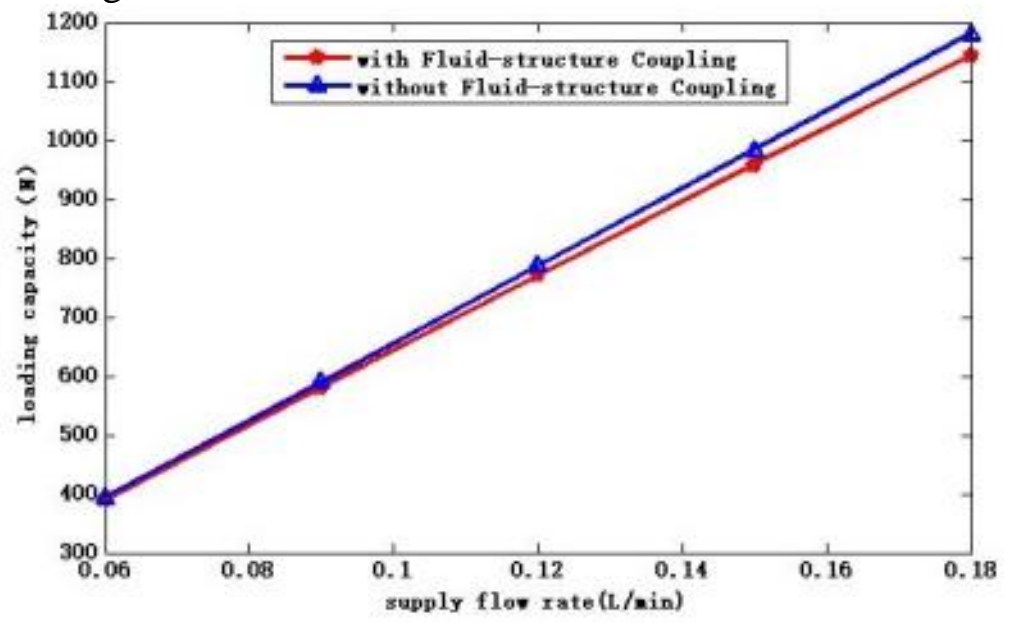

Fig.7 The variation of loading capacity with supply flow rate

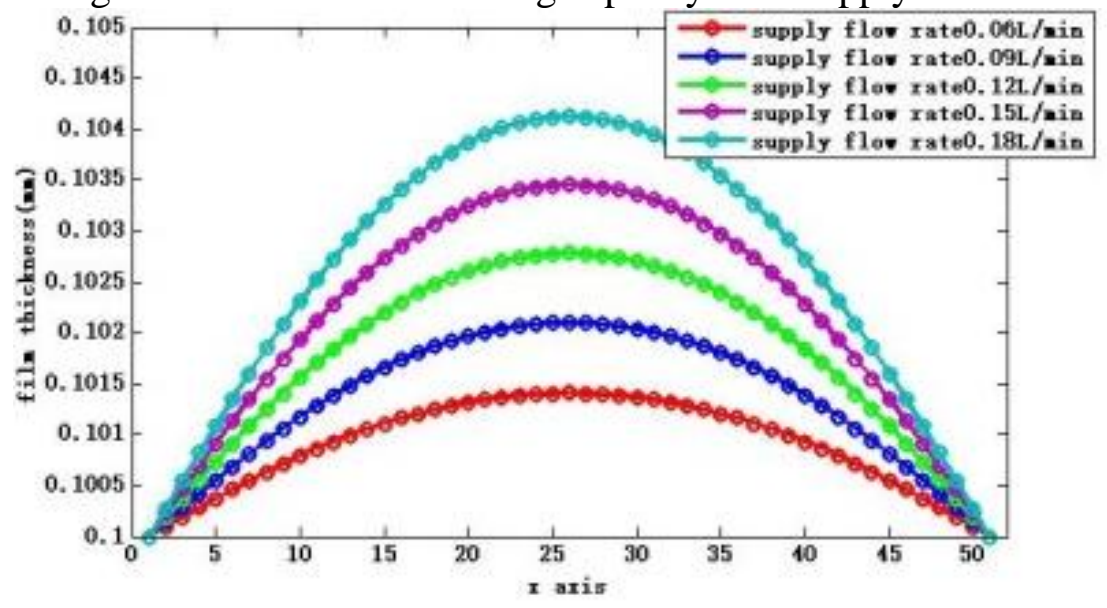

Fig. 8 The variation of railway deformation with supply flow rate

\section{3 railway surface inclination impact on loading capability of single oil pad}

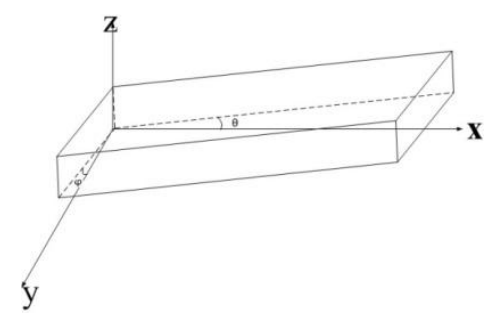

Fig. 9 Schematic diagram of railway surface inclination

The railway surface inclination can be expressed as is shown in Fig. 9. The angle between the railway surface and the $\mathrm{X}$ axis is $\theta$, between the rail surface and $\mathrm{Y}$ axis is $\varphi$. The oil film thickness can be expressed as

$$
\mathrm{h}(\mathrm{i}, \mathrm{j})=h_{0}+h_{x}(i, j)+h_{y}(i, j)
$$

Where $h_{0}$ is the initial oil film thickness, $h_{x}(i, j)$ is the film thickness changing caused by the angle between the railway surface and the $\mathrm{X}$ axis, $h_{y}(i, j)$ is the film thickness changing caused by the angle between the railway surface and the $\mathrm{Y}$ axis.

Fig. 10 shows the pressure distribution with the inclination along the $\mathrm{X}$ axis, while the film thickness and the supply flow rate are constant. Because of the film thickness changing, as is shown in Fig. 11, the loading capacity changes. 


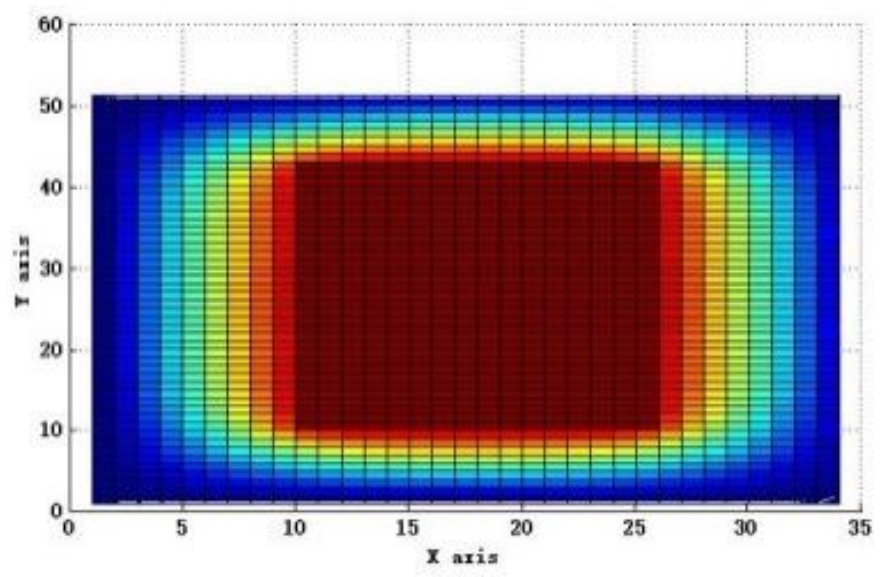

Fig. 10 Pressure distribution diagram of inclination along the $\mathrm{X}$ axis

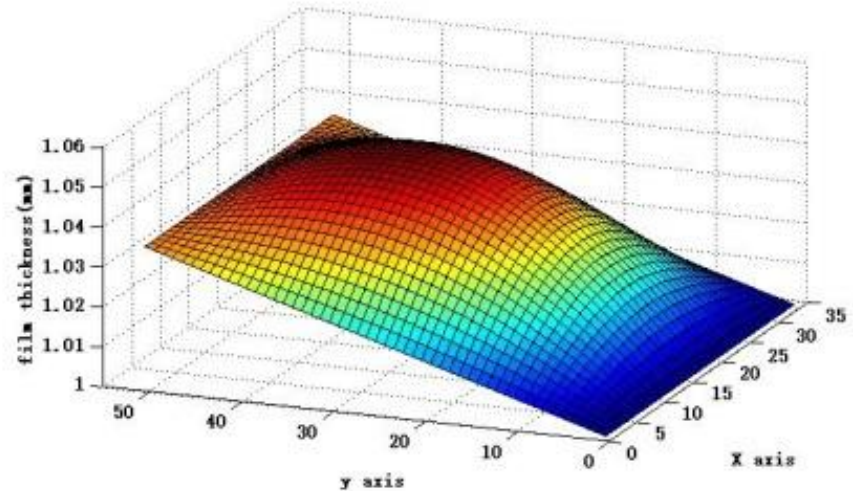

Fig. 11 Film thickness of inclination along the $\mathrm{X}$ axis

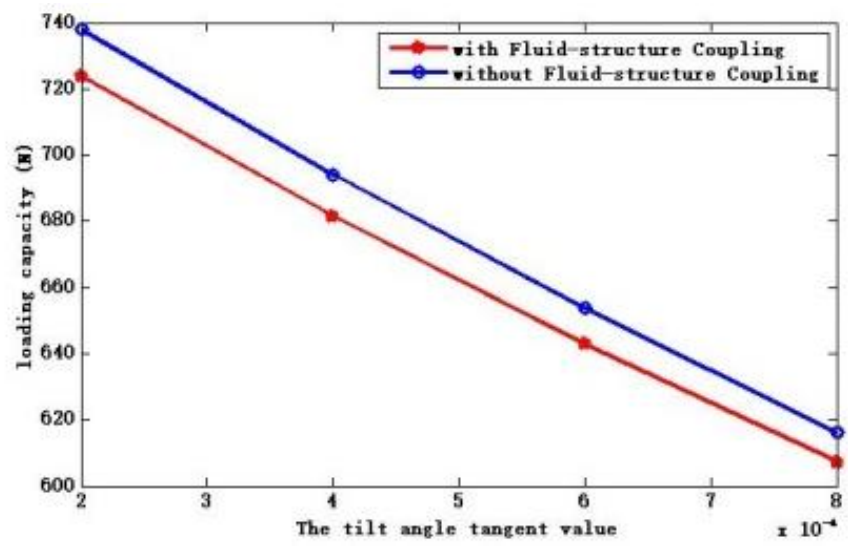

Fig. 12 The variation of loading capacity with $\mathrm{X}$ axis tilting angle

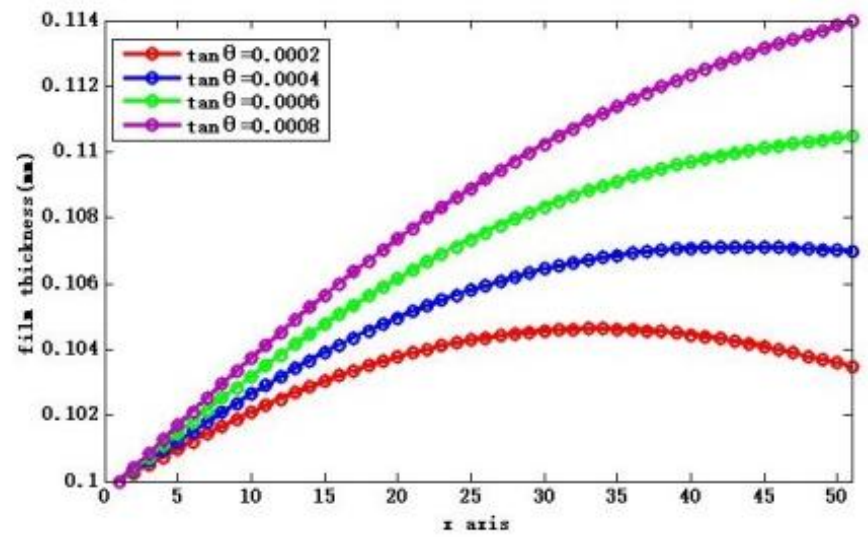

Fig. 13 The variation of film thickness with $\mathrm{X}$ axis tilting angle 
The variation of loading capacity with $X$ axis tilting angle is depicted in Fig. 12 and The variation of film thickness with $X$ axis tilting angle shows in Fig. 13. It is indicated that the loading capacity decreases as the increasing of tilting angle. However the gap between considering the Fluid-structure Coupling and regarding the railway as rigid is almost constant. That is because the supply flow rate is $0.12 \mathrm{~L} / \mathrm{min}$ and the film thickness is $0.1 \mathrm{~mm}$, the deformation caused by the tilting angle is small. It is same trend as the inclination with $\mathrm{Y}$ axis.

\section{Conclusion}

In this paper, an analysis model considering Fluid-structure Coupling is established. The loading capacity of the oil film is studied under different condition. It indicate that compared with regarding the railway as rigid, the loading capacity decreases considering Fluid-structure Coupling. Supply flow rate and the film thickness influence the loading capacity larger compared with the railway surface inclination. Because the deformation caused by the supply flow rate changing and the film thickness changing is greater than railway surface inclination.

\section{Acknowledgements}

This work was supported by National Science and Technology Major Project of China (No.2013ZX04013-011) and National Natural Science Foundation of China (No.51375025), National Science and Technology Major Project of China (No. 2013ZX04013011, 2015ZX04014-021) and Jing-Hua Talents Project of Beijing University of Technology.

\section{References}

[1] N. D. Manring: Journal of Tribology Vol.124 (2002), p.874-877.

[2] Jaw-Ren Lin: International Journal of Machine Tools \& Manufacture Vol.40 (2000), p.1671-1689.

[3] Bouyer J, Fillon M. On the significance of thermal and deformation effects on a plain journal bearing subjected to severe operating conditions. Journal of Tribology, 2004, 126:819-22.

[4] WANG X, YAMAGUCHI A. Characteristics of hydrostatic bearing/seal parts for water hydraulic pumps and motors. Part 1: Experiment and theory[J]. Tribology International, 2002, 35 (7): 425433.

[5] WANG X, YAMAGUCHI A. Characteristics of hydrostatic bearing/seal parts for water hydraulic pumps and motors. Part 2: On eccentric loading and power losses[J]. Tribology International, 2002, 35 (7): 435-442.

[6] KAZAMA T, YAMAGUCHI A. Application of a mixed lubrication model for hydrostatic thrust bearings on hydraulic equipment[J]. ASME Journal of Tribology, 1993, 115(4): 686-691.

[7] KAZAMA T, AMAGUCHI A. Experiment on mixed lubrication of hydrostatic thrust bearings for hydraulic equipment[J]. ASME Journal of Tribology, 1995, 117(3): 399-402.

[8] PANG Zhicheng, ZHAI Wenjie, SHUN Jingwu. The study of hydrostatic lubrication of the slipper in a high-pressure plunger pump[J]. Tribology Transactions, 1993, 36(2): 316-320. 